\title{
COMPARISON BETWEEN DIGITAL SUBTRACTION ANGIOGRAPHY AND MAGNETIC RESONANCE ANGIOGRAPHY IN INVESTIGATION OF NONLACUNAR ISCHEMIC STROKE IN YOUNG PATIENTS
}

\author{
Preliminary results
}

\author{
Adriana Bastos Conforto', Felipe Fregni², Paulo Puglia Jr. ${ }^{3}$, Claudia da Costa Leite ${ }^{3}$, \\ Fabio luji Yamamoto', Karen F. Coracini ${ }^{1}$, Milberto Scaff ${ }^{1}$
}

\begin{abstract}
Purpose: We preliminarily investigated the relevance of perf o rming digital subtraction angiography (DSA) in addition to magnetic resonance angiography (MRA) in definition of ischemic stroke etiology in young patients. Method: DSAs and MRAs from 17 young patients with nonlacunar ischemic stroke were blindly analyzed and their impact on stroke management was evaluated. Results: Etiologies were the same considering results of either DSA or MRA in 12/17 cases. In 15/17 patients no changes would have been made in treatment, regardless of the modality of angiography considered. Conclusion: These preliminary results suggest that DSA may be redundant in two thirds of ischemic strokes in young patients. Further larger prospective studies are necessary to determine indications of DSA in this age group.
\end{abstract}

KEY WORDS: TOAST, stroke etiology, embolism, arterial lesions, cryptogenic stroke.

\begin{abstract}
Comparação entre arteriografia digital e angioressonância na investigação de acidente vascular cerebral isquêmico não-lacunar em pacientes jovens: resultados preliminares

RESUMO - Propósito do estudo: Investigar de forma preliminar a relevância da realização de angiografia digital (AD) adicionalmente a angioressonância (AR) na definição de etiologias de acidente vascular cerebral isquêmico (AVCl) em pacientes jovens. Método: ADs e ARs de 17 pacientes jovens com AVCls nãolacun a resforam analisadas. Avaliamos o impacto destes exames no manejo clínico dos casos. Resultados: Em 12/17 casos, as etiologias dos AVCls de acordo com os resultados de AD ou de AR foram idênticas. Em 15/17 pacientes, nenhuma mudança de conduta terapêutica seria realizada, independentemente da modalidade de exame considerada. Conclusão: Estes resultados preliminares sugerem que os resultados da $A D$ podem ser redundantes em relação à AR em até dois terços dos pacientes jovens com AVCI. Estudos prospectivos maiores são necessários para otimizar o estabelecimento de indicações de $A D$ nesta faixa etária.
\end{abstract}

PALAVRAS-CHAVE:TOAST, etiologia de acidente vascular cerebral, embolia, lesões arteriais, criptogênico.

High incidences of stroke in young individuals have been reported in developing countries ${ }^{1-3}$. In Brazil, it has been estimated that $10.6 \%$ of ischemic strokes a ffect patients aged 15-40 years ${ }^{2}$. In young patients, c ryptogenic strokes account for $8.3-55 \%$ of ischemic st rdkes worldwide, reflecting the challenge of determining stroke mechanisms in this age group ${ }^{1-11}$. While ethnical and regional lifestyle diff e rences are likely to influence incidence and etiological mechanisms of stroke in the young in diff e rent populations, other factors such as use of strict criteria and extent of neurovascular investigation are equally important for diagnosis $^{4,7-9}$. Given that therapeutic decisions depend on stroke etiology, accurate diagnosis is crucial for proper management of these patients.

It is known that characteristics of vascular lesions and etiologies of infarcts are influenced by age ${ }^{4,11}$. Ab n o rmal digital subtraction angiography (DSA) re-

${ }^{1}$ Ne u rology Clinical Division, Hospital das Clínicas, São Paulo University, Brazil (USP); ${ }^{2}$ Beth Israel/Deaconess Medical Center, Harvard Medical School, USA; ${ }^{3}$ Radiology Department, Hospital das Clínicas, USP.

Received 13 September 2005, received in final form 29 December 2005. Accepted 15 February 2006.

Dra. Adriana Bastos Conforto - Neurology Division, Hospital das Clínicas / USP - Av. Dr. Enéas de Carvalho Aguiar 255 / 5084 05403-000 São Paulo SP - Brasil. E-mail: abconf@usp.br 
sults have been reported in $39.6-76 \%$ of young stroke patients $s^{4,9}, 10,12-14$. However, because of concerns about complications of the procedure ${ }^{15}$, DSA has been pro$g$ ressively substituted by magnetic resonance angiography (MRA) for evaluation of cervicocranial arteries in clinical practice. Indeed, good correlations between MRA and DSA have been reported for extracranial and intracranial arterial lesions but studies have focused predominantly on patients older than 45 years ${ }^{16-20}$ or in lacunar strokes in younger patients ${ }^{21}$. Therefore, it remains to be determined whether limitations in MRA accuracy and underperf o rmance of DSA have implications in definition of etiology of nonlacunar stroke in the young and in therapeutic decisions.

We have preliminarily investigated the clinical relevance of performing DSA in addition to MRA in definition of stroke etiology and treatment in a Brazilian sample of patients with ischemic cere b rovascular disease aged less than 45 years.

\section{METHOD}

We reviewed cases of ischemic stroke or transient ischemic attack (TIA) consecutively referred to our neurology ward. The study was part of a larger stroke database protocol approved by the Ethics Committee of Hospital das Clínicas/São Paulo University. The study was retrospective and informed consent was considered unnecessary as long as patients were not identified. Inclusion criteria were: age less than 45 years; performance of intracranial MRA (iMRA), ce nical MRA (CMRA) and DSA. Images that did not fulfil quality criteria were excluded. MRAs and DSAs were reviewed independently by two senior neuroradiologists (PP and $\mathrm{CCL}$ ). Both were blind to clinical data and lesional topographies in cranial magnetic resonance imaging (MRI).

Standard imaging protocols for our institution were used. iMRAs were performed with a 1.5 T system with unenhanced 3D time-of-flight (TOF) multiple overlapping thinslab acquisition (MOTSA) sequences, three-dimensional maximal intensity projection (MIP) images (TR/TE $33.3 / 3 \mathrm{ms,}$ flip angle $20^{\circ}$, thickness $=0.8 \mathrm{~mm}$, matrix $256 \times 192$, field of view from $13 \times 13$ to $22 \times 22 \mathrm{~cm}, 0 \mathrm{~mm}$ interval). cMRAs were performed with contrast-enhanced 3D TOF MOTSA sequences with MIP images (TR/TE 7-23/4.4 ms, flip angle 45-50', thickness $=2.6 \mathrm{~mm}$, matrix $256 \times 160$, field of view $26 \times 26 \mathrm{~cm}$, $0 \mathrm{~mm}$ interval) and a sm art preparation technique. Intraa rterial 4-vessel selective DSA was performed via the femoral artery, starting with imaging of the aortic arch followed by selective injections of contrast material into both carotid and vertebral arteries. DSA (GE Medical Systems) was performed in the anteroposterior, lateral and oblique projections. Non-ionic contrast of low osmolarity (lopamiron ${ }^{\circledR}$, Schering) was administered with a power injector at a rate of $6 \mathrm{~mL} / \mathrm{s}$ in the common carotid and subclavian arteries (volume: $8 \mathrm{~mL}$ ) and a rate of $4 \mathrm{~mL} / \mathrm{s}$ in the internal carotid and vertebral arteries $(6 \mathrm{~mL})$. Hydrophilic coated guide wi- res and 4 or $5-\mathrm{F}$ sheaths were used. Sheaths were intermittently flushed with heparinized saline (5000 IU of heparin in $1000 \mathrm{~mL}$ of normal saline). Manual compression at the puncture site was usually performed for 10 minutes after the end of the procedure. No DSA complications were reported.

A standard protocol was systematically used for analysis of DSAs and MRAs blindly to clinical features, MRI findings and laboratory results. Arterial segments were classified as: normal/stenosis $\leq 50 \%$; stenosis $>50 \%$; occlusion. Final diagnoses were defined based on characteristics of lesions and overall findings. Signs considered suggestive of embolism were: carotid " $\mathrm{T}$ " or basilar tip occlusion; abrupt and isolated occlusion of an intracranial arterial branch in the absence of signs of progressive disease in other arteries. Sources of emboli in proximal vessels were evaluated.

All of the patients underwent clinical and neurological evaluation, electrocardiogram, hemogram, blood biochemistry lipid profile, erythrocyte sedimentation rate, immunologic and coagulation testing, cranial computed tomography and MRI. Other investigations included transesophageal echocardogram (TEE) (16), duplex ultrasonography of extracranial vessels (15), transthoracic echocardiogram (TTE) (7), se rology for syphilis and Chagas' disease (15), cerebrospinal fluid analysis (16), transcranial Doppler (8), Holter monitoring (7). The only patient who underwent TTE but not TEE had a positive serology for Chagas' disease and evidence of cardiomyopathy on TTE. Chagas' disease is caused by Trypanosoma cruzi infection, endemic in some South American regions ${ }^{22}$. Two experienced neurologists (MS and FY) defined by consensus the most likely etiological diagnosis according to TOAST criteria ${ }^{23}$ : large-a rt e ry atherosclerosis, cardioembolism, small-vessel occlusion, stroke of other determined etiology and st roke of undetermined etiology. In addition, when serology for Chagas' disease was positive and there was evidence of cardiomyopathy, the diagnosis was cardioembolism. Results of DSA and MRA were given for each case; neurologists were blind to the modality of angiography and the order of DSA and MRA results was randomized. Treatment of each patient (antiplatelet d rugs/ anticoagulants/ other) was defined, considering clinical and laboratorial investigation as well as results of MRA or DSA. We evaluated agreement between etiology of stroke and agreement between treatment, considering results of either exam.

Given the small sample size of this study (17 patients), this study was underpowered for perf o rmance of statistical tests. Therefore, the two methods of neuroimaging were compared in a descriptive way.

\section{RESULTS}

A total of 35 patients younger than 45 years were admitted between January and October, 2002; 20/35 patients underwent iMRA, CMRA and DSA. Films from 19 patients were available and were photographed. Two cases did not meet quality criteria and were excluded; in one case, only the posterior cerebral arteries did not fulfill quality criteria and were therefore 







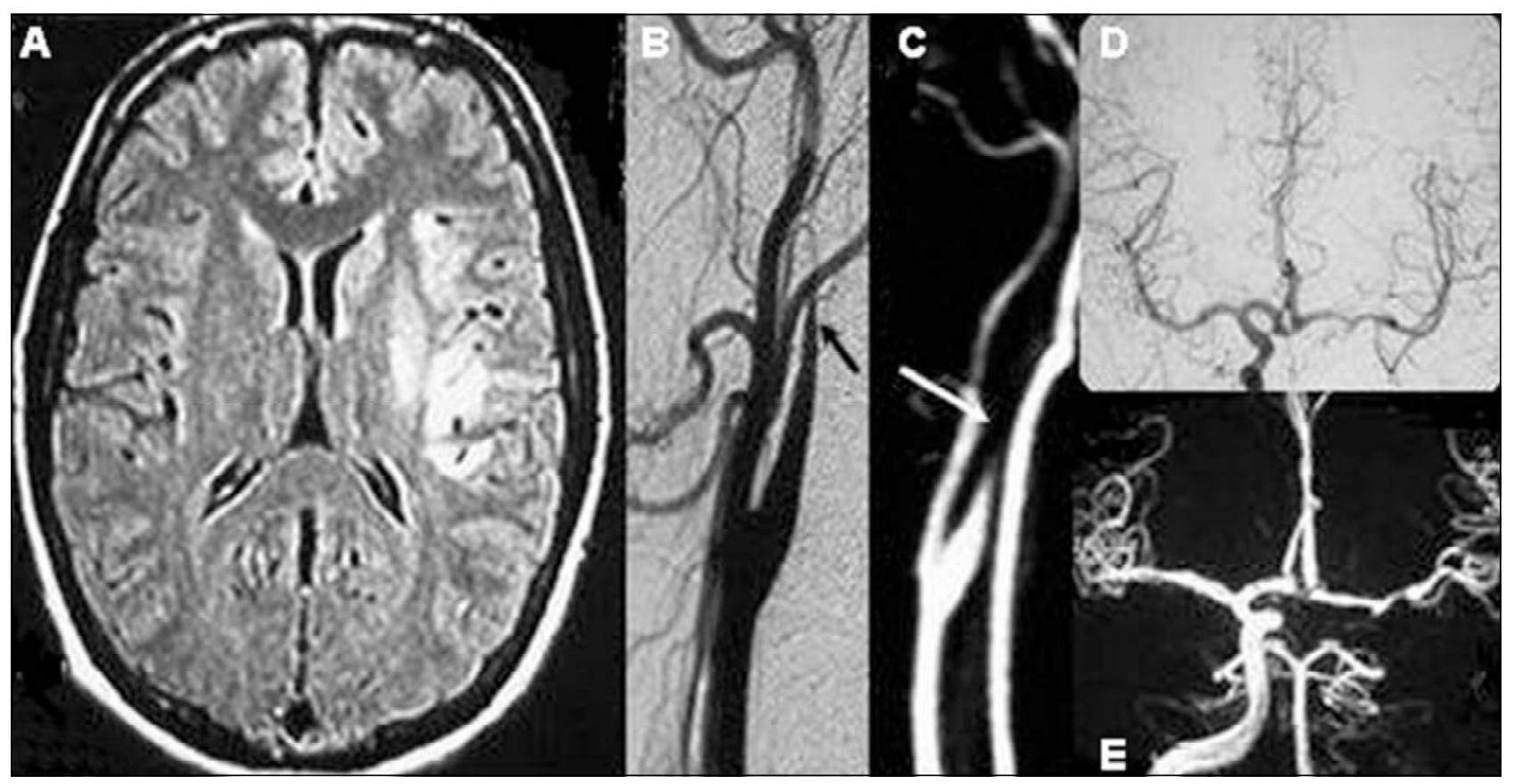

Fig 1. Patient 1, a 26-year-old male, had sudden onset of headache, nausea, right hemiparesis and dysarthria after sexual inter course. (A) Fluid attenuated inversion-recovery(FLAIR) sequence in cranial MRI showed an infarction involving the territory of left lenticulostriate and insular MCA branches. (B) DSA demonstrated left cervical ICA segmental stenosis (arrow) suggestive of arte rial dissection and left intracavernous ICA occlusion. (D) left ACA and MCA were filled by the Willis circle. (C, E) MRA disclosed sim ilar findings.

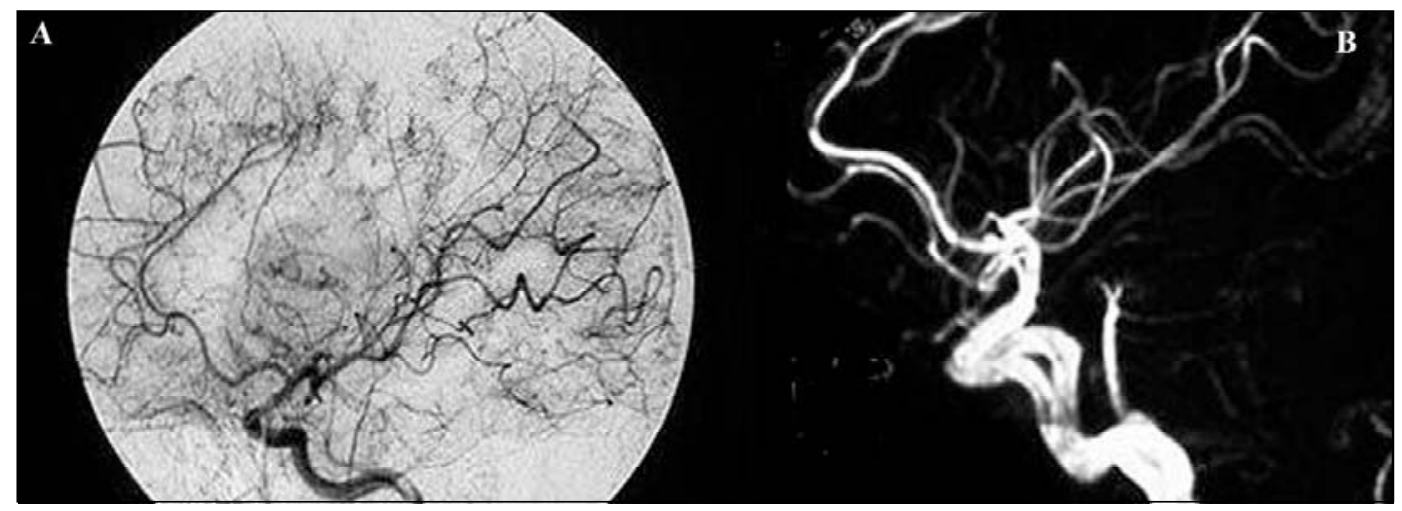

Fig 2. Patient 15, a 29 year-old female, had history of seizures, arthralgias, Raynaud phenomenon and alope cia. She had sudden onset of left monoparesis. DSA showed contrast retention in cortical vessels (A). MRA was normal (B).

not evaluated. Therefore, iMRAs, cMRAs and DSAs from 17 patients aged 17-44 years (mean \pm standard deviation, $33 \pm 9$ years), 8 male, were analyzed.

All DSAs and 12/17 MRAs revealed abnomalities. Clinical features, affected arterial territories, DSA and MRA results as well as diagnosis according to TOAST criteria considering either DSA or MRA results are shown in the Table. None of the infarcts was lacunar. The most frequently affected artery was the middle ce rebral art e ry (MCA) (8/17 in both MRA and DSA) (Table). MRA and DSA final diagnoses were complete- ly concordant in only 4 patients (Table, patients 1,2 , $4,5)$. Fig 1 (A-E) shows images from patient 1 . Intervals between examinations were (mean \pm standard deviation): iMRA and DSA, $3.5 \pm 5.6$ days, cMRA and DSA, $1.4 \pm 7.1$ days.

MRA had lower resolution than DSA for detection of arterial branch lesions (Patients 6-8, 10-13, 15). Fig 2 shows images from case 15. Also, MRA did not reveal a posterior cerebellar inferior art ery (PICA) lesion (patient 3), a lesion of fibromuscular dysplasia (patient 14) and 2 arterial dissections (patients 16 
and 17) (Table). In case 9, DSA disclosed left VA dissection and MRA, left MCA lesions but the patient had a right striatocapsular infarction. There fore, DSA probably showed a false positive result in this case.

In patients 3, 6-8 and 10-13, DSA revealed focal lesions suggestive of embolism in arteries supplying the infarcted area $3,6,7,12$. Yet, emboligenic sources were only detected in patients 11 and 12 .

Etiologies according to TOAST criteria were the same considering results of either DSA or MRA in 12/ 17 cases (Table, patients 1 to 12 ) and were different in 5/17 cases (Table, patients $13-17$ ). In patient 14 , diagnosis was large-artery athero scle rosis based on MRA findings but DSA revealed fibromuscular dysplasia. In patient 13, the diagnosis was large-artery atherosclerosis considering the MRA finding of MCA stenosis but was undetermined considering the absence of large-artery stenosis and the presence of a lesion suggestive of embolism in DSA. In 9/17 cases (patients 5-10, 15-17) etiological diagnosis was undetermined considering MRA results. In 3 out of these 9 cases, DSA contributed significantly to definition of stroke etiology: vasculitis (patient 15) and arterial dissection (patients 16 and 17). In the remaining cases, etiological diagnosis remained undetermined in spite of DSA results.

Neurologists chose different treatments in patients 16 and 17 only (anticoagulation based on DSA results, antiplatelet drugs based on MRA results). In all other 15 cases, no changes would have been made in treatment, regardless of the modality of angiography evaluated.

\section{DISCUSSION}

To our knowledge, this is the first study to dire ctly compare MRA and DSA findings in nonlacunar ischemic stroke in young adults. Concordance between final angiographic diagnoses obtained by different imaging modalities was low. On the other hand, concordance between stroke etiologies according to clinical and imaging criteria was high, as well as concordance between therapeutic decisions based on results of either MRA or DSA.

DSA identified abnormalities in at least one artery in all patients and MRA, in more than two thirds of the patients. It has been reported that lacunar strokes are highly predictive of normal DSAs in young patients with ischemic stroke ${ }^{21}$. None of the strokes in our series was lacunar (Table) and this is a likely reason to explain the high rate of abnormal DSAs in the present series.
Occlusion suggestive of embolism has been described as the most frequent DSA abnormality in young stroke patients ${ }^{12,14}$. In this series, embolism to intracranial arteries may have been an important etiology of stroke. First, the most commonly affected artery was the MCA, an usual target for emboli. Second, DSA revealed lesions suggestive of embolism $^{3,6,7,12}$ in arterial branches in 8 cases (Patients 3, 68 and 10-13). In 4 of these cases the lesions were not detected in MRA. In addition, in patient 15 lesions in cortical areas were demonstrated by DSA only. These findings may be explained by lower MRA resolution for visualization of terminal carotid branches and intracranial vessels ${ }^{24}$.

The detection of intracranial branch arterial lesions in DSA in the absence of identifiable sources of embolism in the present series supports the view that underdiagnosed embolism may be partially responsible for a higher rate of cryptogenic strokes in younger individuals. Other authors have described angiographic findings suggestive of embolism in the absence of identifiable sources in stroke of undetermined cause $^{6,25}$. Some thrombi may lyse from their sources or may be too small to be detected by current tools of investigation ${ }^{26,27}$. Therefore, quoting L.R. Caplan, it is possible that DSA may have revealed the "birds" while their "nests" remained unknown in some of the presented cases $^{28}$.

However, in order to consider embolism as the etiology of an infarction, it is crucial to identify an emboligenic source. In patients 11 and 12, emboligenic sources were determined and stroke etiology would be cardiac embolism regardless of the modality of angiography considered. In 5 of 8 patients (Table, cases 6-8, 10 and 13), DSA revealed lesions suggestive of embolism but no emboligenic sources we re found and causes of stroke remained undetermined. These results indicate that differences in MRA and DSA sensitivity for detection of lesions suggestive of embolism did not substantially influence the final etiological diagnosis in these patients.

A critical finding was the concordance between diagnoses of stroke etiology according to TOAST criteria in two thirds of the cases, given the results of either MRA or DSA. Treatment would have been diffe rent in only two cases, both arterial dissections demonstrated by DSA. False negative results in MRA have been previously reported in arterial dissection ${ }^{29}$. Cervical MRI with axial T1 or T2-weighted images with fat suppression increases sensitivity to dissection by identification of the hematoma in the arte- 
rial wall ${ }^{30}$ and was not performed in the two patients because of lack of clinical suspicion at the time they were evaluated. If MRI had shown intramural hematomas in the dissected arteries, no differences in diagnosis would have occurred considering MRA/MRI or DSA findings and therefore, no differences in treatment would have been made for any of the patients.

Technical improvements contributed to decre ase the risk of neurologic complications reported in the literature ${ }^{31,32}$ : Wilinsky and colleagues re ported 1.3\% ne u rologic complications in 2,899 cerebral angiographies $^{15}$. Complications were transient in $0.2 \%$, reversible in $0.7 \%$ and permanent in $0.5 \%$ of the cases. Age greater than 55 years, presence of cardiovascular disease and fluoroscopic times of 10 minutes significantly increased risk associated with DSA. It is still a matter of controversy whether ischemic stroke inc reases DSA risk, compared to other conditions such as subarachnoid hemorrhage ${ }^{15}$.

DSA may be safer in younger patients without diffuse vasculopathy than in older individuals ${ }^{12,15}$. However, risk-benefit ratios should be considered and invasive procedures should be avoided whenever additional information does not substantially contribute to definition of diagnosis and treatment. Therefore, DSA in stroke in the young must be more clearly defined. These preliminaryfindings emphasize the need to enhance identification of sources of embolism and the importance of an integrated clinical/radiological a p p roach. Due to small sample size of this study, we performed a descriptive analysis of the data. Therefore, we do not know if the results of this study may be externally generalized. However, the findings of this study encourage future prospective studies involving larger number of patients to determine in which cases DSA is more likely to have a relevant impact on definition of ischemic stroke etiology and treatment in young patients.

\section{REFERENCES}

1. Radhakrishnan K, Ashok PP, Sridharan R, Mousa ME. Stroke in the young: incidence and pattern in Benghazi, Lybia. Acta Neurol Scand 1986;73:434-438.

2. Siqueira JI Neto, Santos AC, Fabio SR, Sakamoto AC. Cerebral infarction in patients aged 15 to 40 years. Stroke 1996;27:2016-2019.

3. Awada A. Stroke in Saudi Arabian young adults: a study of 120 cases. Acta Neurol Scand 1994;89:323-328.

4. Adams HP Jr., Kappelle LJ, Biller J, et al. Ischemic stroke in young adults: experience in 329 patients enrolled in the Iowa Registry of stroke in young adults. Arch Neurol 1995;52:491-495.

5. Bogousslavsky J, Pierre P. Ischemic stroke in patients under age 45 . Neurol Clin 1992;10:113-124.

6. Kristensen B, Malm J, Carlberg B, et al. Epidemiology and etiology of ischemic stroke in young adults aged 18 to 44 years in northern Sweden. Stroke 1997;28:1702-1709.

7. Jacobs BS, Boden-Albala B, Lin IF, Sacco RL. Stroke in the young in the northern Manhattan stroke study. Stroke 2002;33:2789-2793.
8. Chan MT, Nadareishvili ZG, Norris JW. Diagnostic strategies in young patients with ischemic stroke in Canada. Can J Neurol Sci 2000;27:120124.

9. Carolei A, Marini C, Ferranti E, Frontoni M, Prencipe M, Fieschi C. A prospective study of cerebral ischemia in the young: analysis of pathogenic determinants. The National Research Council Study Group. Stroke 1993;24:362-367.

10. Kwon SU, Kim JS, Lee JH, Lee MC. Ischemic stroke in Korean young adults. Acta Neurol Scand 2000;101:19-24.

11. Musolino R, La Spina P, Granata A, et al. Ischaemic stroke in young people: a prospective and long-term follow-up study. Cerebrovasc Dis 2003;15:121-128.

12. Smoker WR, Biller J, Hingtgen WL, Adams HP Jr., To ffol GJ. Angiography of nonhemorrhagic cerebral infarction in young adults. Stroke 1987;18:708-711.

13. Stillman MJ, Ronthal M, Kleefield J, et al. Cerebral infarction: shortcomings of angiography in the evaluation of intracranial cerebrovascular disease in 25 cases. Medicine (Baltimore) 1987;66:297-308.

14. Lisovoski F, Rousseaux P. Cerebral infarction in young people: a study of 148 patients with early cerebral angiography. J Neurol Neurosurg Psychiatry 1991;54:576-579.

15. Willinsky RA, Taylor SM, TerBrugge K, Farb RI, Tomlinson G, Montanera W. Neurologic complications of cerebral angiography: p rospective analysis of 2,899 procedures and review of the literature. Radiology 2003;227:522-528.

16. Cosottini M, Pingitore A, Puglioli M, et al. Contrast-enhanced threedimensional magnetic resonance angiography of atherosclerotic internal carotid stenosis as the noninvasive imaging modality in revascularization decision making. Stroke 2003;34:660-664.

17. Phan T, Huston J, 3rd, Bernstein MA, Riederer SJ, Brown RD Jr. Contrast-enh anced magnetic resonance angiography of the cervical vessels: experience with 422 patients. Stroke 2001;32:2282-2286.

18. Nederkoorn PJ, van der Graaf Y, Hunink MG. Duplex ultrasound and magnetic resonance angiography compared with digital subtraction angiography in carotid artery stenosis: a systematic review. Stroke 2003;34:1324-1332.

19. Korogi $Y$, Takahashi M, Mabuchi N, et al. Intracranial vascular stenosis and occlusion: diagnostic accuracy of three-dimensional, Fourier transform, time-of-flight MR angiography. Radiology 1994;193:187-193.

20. Willinek WA, von Falkenhausen M, Born M, et al. Noninvasive detection of steno-occlusive disease of the supra-aortic arteries with threedimensional contrast-enhanced magnetic resonance angiography: a p rospective, intra-individual comparative analysis with digital subtraction angiography. Stroke 2005;36:38-43.

21. De Jong S, Lodder S, Luijckx GJ. Is cerebral angiography redundant in undetermined cause of stroke in patients below 50 years when the stroke is lacunar? J Neurol Sci 2004;222:83-85.

22. C a rod-Artal FJ, Va rgas AP, Melo M, Horan TA. American trypanosomiasis (Chagas' disease): an unrecognised cause of stroke. J Neurol Neurosurg Psychiatry 2003;74:516-518.

23. Adams HP, Jr., Bendixen BH, Kappelle LJ, et al. Classification of subtype of acute ischemic stroke: definitions for use in a multicenter clinical trial. TOAST. Trial of Org 10172 in Acute Stroke Treatment. Stroke 1993;24:35-41.

24. Wutke R, Lang W, Fellner C, et al. High-resolution, contrast-enhanced magnetic resonance angiography with elliptical centric k-space ordering of supra-aortic arteries compared with selective X-ray angiography. Stroke 2002;33:1522-1529.

25. Sacco RL, Ellenberg JH, Mohr JP, et al. Infarcts of undetermined cause: the NINCDS Stroke Data Bank. Ann Neurol 1989;25:382-390.

26. Warach S, Li W, Ronthal M, Edelman RR. Acute cerebral ischemia: evaluation with dynamic contrast-enhanced MR imaging and MR angiography. Radiology 1992;182:41-47.

27. Babikian VL, Caplan LR. Brain embolism is a dynamic process with variable characteristics. Neurology 2000;54:797-801.

28. Caplan LR. Of birds and nests and brain emboli. Rev Neurol (Paris) 1991;147:265-273.

29. Khan R, Smith JK, Castello M. False-negative contrast MRA in the setting of carotid artery dissection. Emerg Radiol 2002;9:320-322.

30. Ozdoba C, Sturzenegger M, Schroth G. Internal carotid artery dissection: MR imaging features and clinical-radiologic correlation. Neuroradiology 1996;199:191-198.

31. Melaragno R. Lateral syndrome of the medulla oblongata (Wallenberg's syndrome) as a complication of a vertebral angiography: a case report. Arq Neuropsiquiatr 1972;30:78-83.

32. Lynch JC, Pereira A. Cegueira cortical transitória após angiografia cerebral. Arq Neuropsiquiatr 1984;42:298-301. 